\title{
Microthermal measurements of surface layer seeing at Devasthal site
}

\author{
P. Pant, C.S. Stalin, and R. Sagar \\ U. P. State Observatory, Manora Peak, Nainital 263 129, India
}

Received August 28; accepted October 21, 1998

\begin{abstract}
In order to detect the microthermal fluctuations introduced by the atmospheric turbulence very near to the ground at Devasthal site, a PC based instrumentation has been developed. The optical image degradation due to such turbulence has been quantified. The results of the optical seeing due to the surface layer at Devasthal site are presented and compared with the seeing results obtained from the Differential Image Motion Monitor. Microthermal measurements were taken on 20 nights between March and June 1998, using sensors placed at three equally spaced levels on a $18 \mathrm{~m}$ high mast. We found a significant decrease in the optical turbulence over the height of the mast with a mean value of $0.32^{\prime \prime}$ for the 12 to $18 \mathrm{~m}$ slab and $1.28^{\prime \prime}$ for the 6 to $12 \mathrm{~m}$ slab. For Devasthal site, a seeing of $\sim 0.6^{\prime \prime}$ can be achieved, if the telescope is located at a height of $\sim 13 \mathrm{~m}$ above the ground.
\end{abstract}

Key words: site atmospheric effects — site testing

\section{Introduction}

Based on several years of meteorological data, Devasthal about $50 \mathrm{~km}$ east of Nainital in India (latitude $=29^{\circ}$ $22^{\prime} \mathrm{N}$, longitude $=79^{\circ} 41^{\prime} \mathrm{E}$, altitude $=2420 \mathrm{~m}$ above mean sea level) has been found as a potential site for putting up a 3-metre class optical telescope jointly by Tata Institute of Fundamental Research (TIFR), Mumbai and U. P. State Observatory (UPSO), Nainital. For characterizing this site astronomically, an intensive site testing campaign is being carried out using as many modern site testing instruments as possible. For the measurement of seeing, two Differential Image Motion Monitors (DIMMs) mounted on both $52 \mathrm{~cm}$ and $38 \mathrm{~cm}$ reflector telescopes located at two different sites of Devasthal are already in use, whilst another instrument for detecting the surface layer microthermal fluctuations and for estimating their contribution to the seeing has been developed and recently

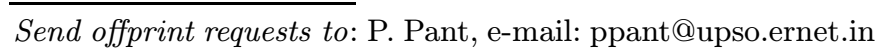

been installed near the $52 \mathrm{~cm}$ telescope site. Broadly, the aim of this study is to evaluate image degradation due to induced atmospheric turbulence within a height of a few metres above the ground. This information can be used to decide the height for locating the telescope in order to achieve better seeing conditions. For this, we started measurements of the turbulence due to surface layer since March 1998.

It is an established fact that atmospheric turbulence with its associated random refractive index inhomogeneities disturbs a passing light beam (Tatarski 1961; Hufnagel \& Stanley 1964; Fried 1966) and in consequence deteriorates the optical quality of the star image. Since the earth surface acts as a heat source during day and as a heat sink during night, the magnitude of microthermal fluctuations within turbulent layers are usually maximum near the ground (Erasmus \& Thompson 1986). When an electromagnetic wave propagates through a refractively nonuniform medium, fluctuations in the amplitude and phase of the wave are introduced. Consequently, an optical image formed by focusing such a wave exhibits fluctuation in intensity, sharpness and position which are termed as scintillation, image blurring and image motion respectively (Coulman 1985). Seeing effects due to small scale temperature gradients have also been studied by using temperature probes inside the telescope dome and in the immediate vicinity of the telescope site (Lynds 1963; Hall 1967; Coulman 1969, 1974). Lynds (1963) estimated the visual image quality with the $90 \mathrm{~cm}$ reflector telescope and compared these observations with the simultaneously taken temperature fluctuation traces. These observations showed that the average value of the seeing was correlated directly with the amplitude and frequency of occurrence of the temperature fluctuations. He also concluded that seeing was never good when the temperature fluctuations were large and frequent. Likewise when the temperature fluctuations were small and infrequent the seeing was generally good.

This paper presents the results of the observations taken on 20 nights during March to June 1998, using 
microthermal sensor pairs placed at three equally spaced levels on a $18 \mathrm{~m}$ high mast. The results have been compared with the seeing data obtained from DIMM instrument mounted on the $52 \mathrm{~cm}$ reflector telescope installed at a height of $\sim 3 \mathrm{~m}$ above the ground in the vicinity of the mast. The seeing measurements from these two different types of data have been analyzed for an understanding of the seeing quality of the Devasthal site.

The next section describes the details of the principle of seeing measurements in terms of optical turbulence. In Sect. 3, there is a brief account of the instrumental design and data analysis. In Sect. 4, the results and discussions are given followed by the conclusions in Sect. 5 .

\section{Principle of seeing measurements}

The correlation between the astronomical seeing and the atmospheric turbulence has been investigated by various authors (Barletti et al. 1974, 1976; Ken Knight et al. 1977; Marks et al. 1996). They have measured the small scale temperature structure functions in order to evaluate the refractive index structure constants for atmospheric layers. These refractive index structures have widely been used for calculating the astronomical seeing quality. Their investigations reveal that the parameter which gives a measure of the optical turbulence intensity related to the refractive index inhomogeneities is the refractive index structure coefficient $C_{\mathrm{N}}^{2}$ (Coulman 1969; Vernin \& MuñozTunón 1994), which is a measure of the average variability of the refractive index of light in the atmosphere (Erasmus \& Thompson 1986). The parameter $C_{\mathrm{N}}^{2}$ is connected with the temperature structure coefficient $C_{\mathrm{T}}^{2}$ of the microthermal field variations, which produce fluctuations in the refractive index at optical wavelengths (Coulman 1969; Barletti et al. 1974). The relationship between refractive index fluctuations $\left(C_{\mathrm{N}}^{2}\right)$ and thermal irregularities $\left(C_{\mathrm{T}}^{2}\right)$ at height $h$ is given by

$C_{\mathrm{N}}^{2}(h)=\left(\frac{8010^{-6} \times P(h)}{T^{2}(h)}\right)^{2} C_{\mathrm{T}}^{2}(h)$

where $P(h)$ and $T(h)$ are the pressure in millibar (mb) and the absolute temperature in Kelvin $(\mathrm{K})$ respectively at height $h$ in metre. Hence the knowledge of $C_{\mathrm{T}}^{2}(h)$ as a function of altitude is prerequisite for the estimation of the astronomical seeing quality of any place. Following Barletti (1974) and Marks et al. (1996), the process for evaluating $C_{\mathrm{T}}^{2}(h)$ involves the measurement of the temperature function $D_{\mathrm{T}}(r, h)$ at points $P_{1}$ and $P_{2}$ at same level $h$, but horizontally separated by a distance $r$ given by

$D_{\mathrm{T}}(r, h)=<\left[T\left(P_{1}\right)-T\left(P_{2}\right)\right]^{2}>$

where, $T(P)$ is the temperature at point $P$ and angle brackets denote the ensemble average. As defined by Obukhov (1949) this is related to $C_{\mathrm{T}}^{2}(h)$ by

$D_{\mathrm{T}}(r, h)=C_{\mathrm{T}}^{2}(h) r^{2 / 3}$
In our case, measuring the temperature differences at two points which are horizontally separated by one metre apart, the value of $D_{\mathrm{T}}(r, h)$ is numerically equal to the $C_{\mathrm{T}}^{2}(h)$ value in $\mathrm{K}^{2} \mathrm{~m}^{-2 / 3}$ units. The relationship between $r_{0}$, the Fried's parameter and $C_{\mathrm{N}}^{2}$ as a function of height $h$ through the atmosphere has been given by Fried (1966) as

$r_{0}=\left(16.7 \lambda^{-2} \int_{0}^{\infty} C_{\mathrm{N}}^{2}(h) \mathrm{d} h\right)^{-3 / 5}$

where $\lambda$ is the wavelength and $C_{\mathrm{N}}^{2}$ is the refractive index structure constant, which gives a measure of the optical turbulence intensity related to the refractive index inhomogeneities in the atmosphere at height $h$. Vernin \& Muñoz-Tuñón (1992) have mentioned that each turbulent layer at its altitude contributes to the degradation of the image according to the intensity of the turbulence. Thus $C_{\mathrm{N}}^{2}$ represents the sum of the contributions from all turbulent layers in the atmosphere. From the theory of wave propagation in turbulent media given by Tatarski (1961) and its relevant application to the astronomical seeing quality (Roddier 1981; Coulman 1985) the relationship between seeing $\left(\epsilon_{\mathrm{fwhm}}\right)$ and $r_{0}$ is given by Dierickx (1992) as

$\epsilon_{\mathrm{fwhm}}=0.98 \frac{\lambda}{r_{0}}$

where $r_{0}$ represents the diameter of the telescope aperture for which diffraction limited image resolution is equal to the full width at half maximum (fwhm) of the seeing limited image. Thus, $r_{0}$ takes into account all the different turbulent layers encountered by the light beam before reaching the ground (Vernin \& Muñoz-Tuñón 1992). Using expressions (4) and (5), it is possible to write seeing as a function of $C_{\mathrm{N}}^{2}(h)$ as

$\epsilon_{\mathrm{fwhm}}=5.25 \lambda^{-1 / 5}\left(\int_{0}^{\infty} C_{\mathrm{N}}^{2}(h) \mathrm{d} h\right)^{3 / 5}$

The refractive index structure constant in this case represents the sum of the contribution from all turbulent layers in the atmosphere (Marks et al. 1996). Vernin \& MuñozTunón (1992) have mentioned that, in order to assess the quality of an astronomical site, it is not sufficient to measure only the optical turbulence integrated over the whole atmosphere but also to evaluate the relative contribution to the smearing of the image from each intervening slab. This information is valuable for deciding height of the telescope location above the ground level in order to obtain better angular resolution images. The turbulence contributions to seeing $\left(\epsilon_{\mathrm{fwhm}}\right)$ originating from different layers is given by

$\epsilon_{\mathrm{fwhm}}($ total $)=\left(\sum_{i} \epsilon_{i}^{5 / 3}\right)^{3 / 5}$

where $\epsilon_{i}$ is the seeing contributed by $i^{\text {th }}$ turbulent layer. 


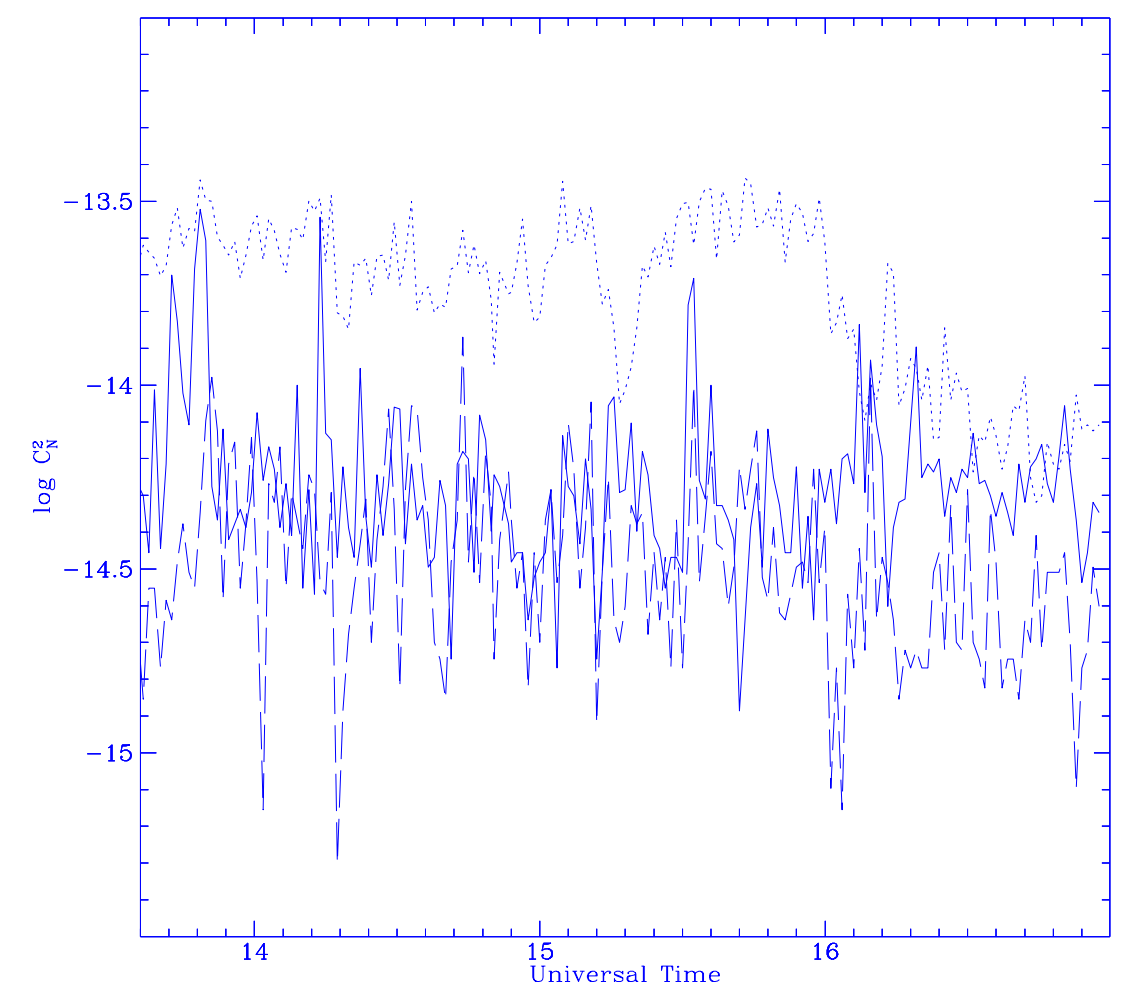

Fig. 1. Temporal evolution of $C_{\mathrm{N}}^{2}$ during the night of 03 May, 1998. Dots represent the $6 \mathrm{~m}$ level, solid line represents the $12 \mathrm{~m}$ level and long dashes the $18 \mathrm{~m}$ level

\section{Instrumental set up and data analysis}

The details of the present experiment have earlier been described by Pant \& Sagar (1998). For recording the microthermal fluctuations at a level, pair of thermal sensors made of thin ( $25 \mu$ in diameter) Nickel wire is used. The horizontal separation between the two sensors is one metre. They are mounted at heights of 6,12 and $18 \mathrm{~m}$ above the ground on the mast. Thus, the inter-spacing between the microthermal sensors is $\sim 6 \mathrm{~m}$. The length of a sensorwire is chosen in such a way that its effective resistance comes out to be $\sim 250 \mathrm{ohm}$ at room temperature. This yields a temperature resolution of $\sim 0.01^{\circ} \mathrm{C}$ for the system. For recording the instantaneous ambient temperature around the microthermal sensors with an accuracy of $0.1^{\circ} \mathrm{C}, \mathrm{Pt}-100 \mathrm{RTD}$ temperature sensor and ADAM4013 analog input module are used. Analog input module ADAM-4013 uses a microprocessor controlled integrating A/D converter to convert RTD signals into digital format. The data are recorded in a PC with the help of the ADAM series of analog input modules and immediately processed in the PC. The ADAM series is a set of intelligent sensor-to-computer interface modules, containing built in micro-controller yielding 16-bit resolution in A/D conversion. They are remotely controlled. The commands are transmitted in RS-485 protocol. The signals from the sensors and RTDs are connected to the electronic circuit inside the observing room by cables. A DC current is fed through these sensors whose resistance changes according to microthermal fluctuations and consequently, produces variable voltage signal for differential amplifier. The amplifier output is sent to a signal processing equipment. The GENIE software package was used for data acquisition, processing and control. Atmospheric pressure is almost the same within a height of $18 \mathrm{~m}$. Its observed value at Devasthal is $\sim 810 \pm 2 \mathrm{mb}$ which has been used in our further data analysis.

\section{Results and discussions}

Microthermal data were successfully recorded on a total of 20 nights during March 1998 - June 1998. The values of $C_{\mathrm{T}}^{2}$ were measured every second and averaged over one minute. These data were used to evaluate the value of $C_{\mathrm{N}}^{2}$. It decrease sharply but nonlinearly with altitude. The seeing contribution due to a slab of turbulent layers is evaluated using Eq. (6) by assuming a power law variation for $C_{\mathrm{N}}^{2}$ within a slab. The height of the slabs range from 6 to $12 \mathrm{~m}$ and 12 to $18 \mathrm{~m}$ above the ground.

Temporal evolution of $C_{\mathrm{N}}^{2}$ on 03 May, 1998 during 13.6 - $17.0 \mathrm{UT}$ at each of the three levels has been illustrated in Fig. 1. This shows that the microthermal turbulence decreased rapidly from 6 to 18 metre height. The average seeing for the lower $(6-12 \mathrm{~m})$ and upper $(12-18 \mathrm{~m})$ slabs are $1.08^{\prime \prime}$ and $0.31^{\prime \prime}$ respectively. 


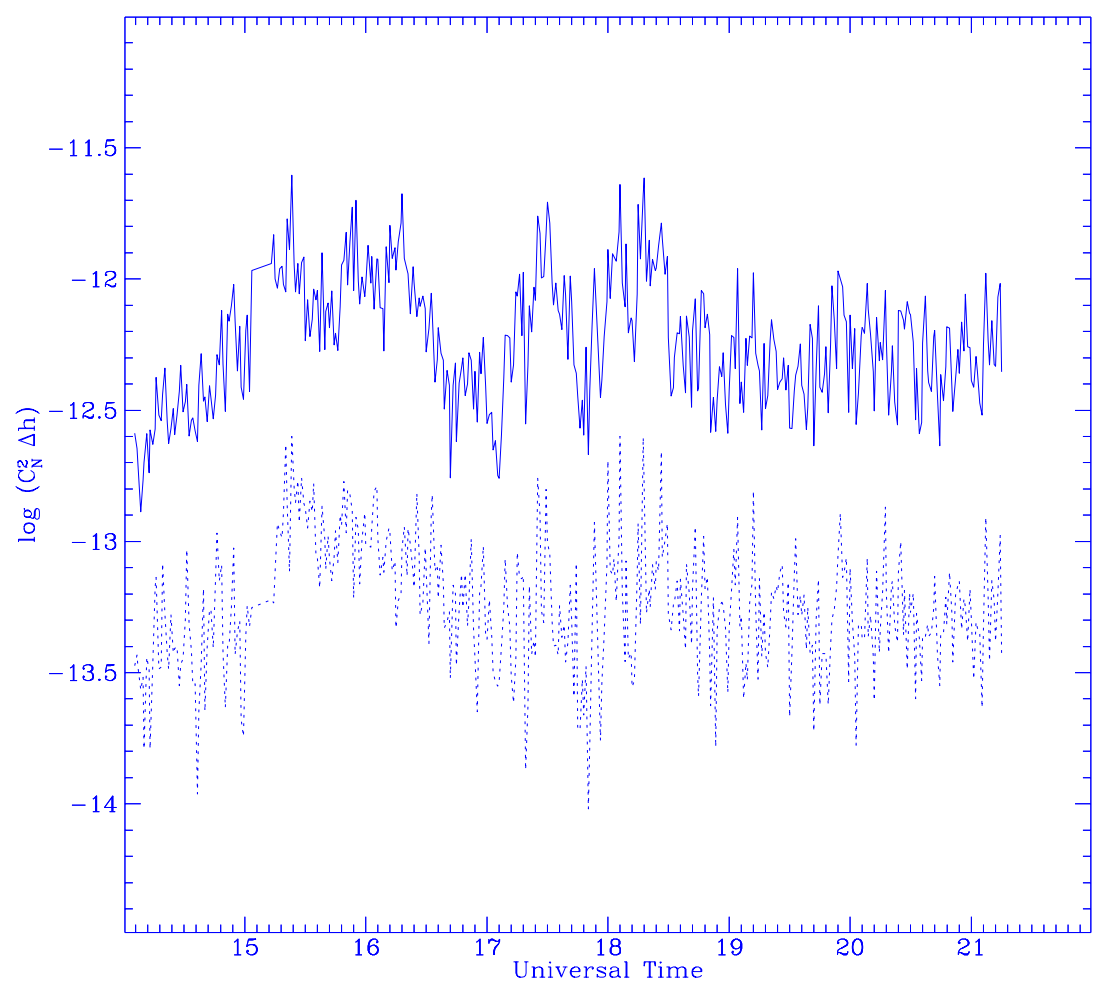

Fig. 2. Turbulence in the surface layer measured from microthermal sensors on the $18 \mathrm{~m}$ high mast on 05 June, 1998 . The solid line gives the $6-12 \mathrm{~m}$ contribution whereas the dots refer to the $12-18 \mathrm{~m}$ contribution

In Fig. 2, the derived values of $C_{\mathrm{N}}^{2} \Delta h$ for 05 June, 1998 are plotted against time in UT for the above mentioned two slabs. The upper curve indicates the turbulence contribution between 6 and $12 \mathrm{~m}$ whereas the lower curve indicates the contribution from 12 to $18 \mathrm{~m}$ slab above the ground. These plots also show that the significant contribution of microthermal fluctuations due to turbulence comes from the lower slab.

Figures $3 \mathrm{a}$ and $3 \mathrm{~b}$ illustrate the statistics of seeing derived from both the microthermal and the DIMM measurements. The frequency and the cumulative distributions of the seeing due to the two slabs are shown in Fig. 3a. The mean and median values of the seeing due to 6 to $12 \mathrm{~m}$ slab are $1.28^{\prime \prime}$ and $1.17^{\prime \prime}$ respectively. The corresponding values due to the 12 to $18 \mathrm{~m}$ slab are almost identical $0.32^{\prime \prime}$ and $0.30^{\prime \prime}$ respectively. The attained values are remarkable. The seeing is better than $0.5^{\prime \prime}$ for $88 \%$ of the time, while it is better than $0.3^{\prime \prime}$ for $50 \%$ of the time, with minimum values around $0.1^{\prime \prime}$. Figure $3 \mathrm{~b}$ shows the frequency and cumulative distributions of the seeing obtained from the DIMM measurement and of the seeing contribution derived for the 6 to $18 \mathrm{~m}$ slab. The mean and median values for the 6 to $18 \mathrm{~m}$ slab are $1.36^{\prime \prime}$ and $1.25^{\prime \prime}$ respectively. The corresponding values for DIMM measurements are $1.51^{\prime \prime}$ and $1.42^{\prime \prime}$ respectively. The results of these measurements are tabulated in Table 1.

In order to have a better insight into the atmospheric behaviour which gives rise to the optical turbulence, the
Table 1. The mean and median values of microthermal and DIMM seeing measurements at Devasthal site during March to June 1998

\begin{tabular}{ccc}
\hline $\begin{array}{c}\text { Height range } \\
\text { (metre) }\end{array}$ & $\begin{array}{c}\epsilon(\text { mean }) \\
\text { (arcsec) }\end{array}$ & $\begin{array}{c}\epsilon \text { (median) } \\
\text { (arcsec) }\end{array}$ \\
\hline 6 to 12 & 1.28 & 1.17 \\
12 to 18 & 0.32 & 0.30 \\
6 to 18 & 1.36 & 1.25 \\
DIMM & 1.51 & 1.42 \\
\hline
\end{tabular}

data were analyzed for the temporal evolution of the seeing over two slabs 6 to $12 \mathrm{~m}$ and 12 to $18 \mathrm{~m}$ and compared with the DIMM measurements. Figure 4 illustrates the temporal variation of the seeing deduced from both microthermal and the DIMM measurements on 02 May, 1998. This and Table 1 show that significant contribution of the thermal deterioration of the optical seeing comes from the 6 to $12 \mathrm{~m}$ slab. The thermal disturbances affect this layer most as compared to the higher layers since it is located very near to the ground. For astronomers, it is desirable to have a characterization of the temporal evolution of the seeing i.e., to know the typical time interval of seeing variation and also the dependence of seeing quality with time (Muñoz-Tuñón et al. 1997). While examining individual nights we do not find any general trend in the seeing evolution as has been found by Muñoz - Tunón et al. (1997) for the ORM site. This is in contrast to the general 

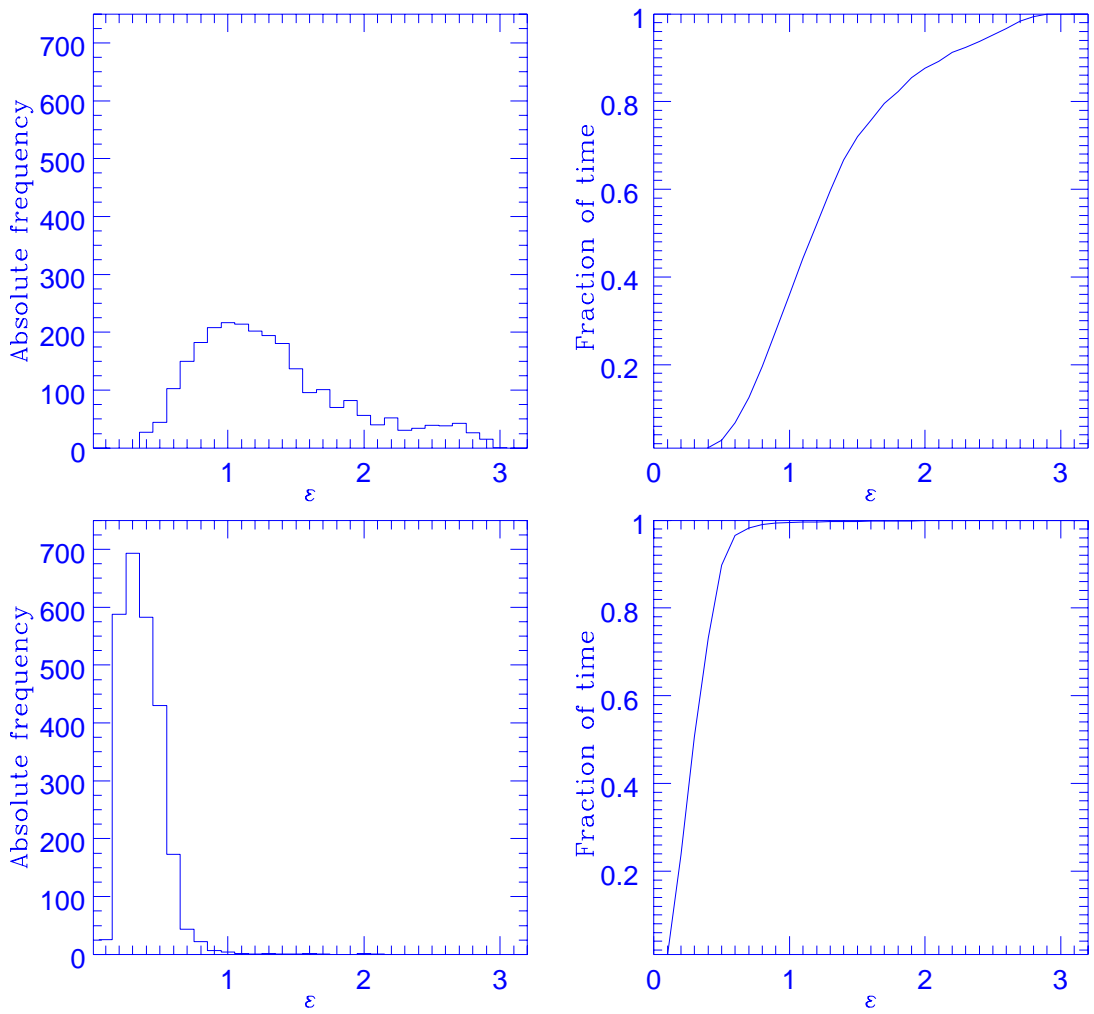

Fig. 3a). Seeing data statistics: FWHM probability distribution and associated cumulative distribution function for two separate levels i.e. $6-12 \mathrm{~m}$ (upper plot) and $12-18 \mathrm{~m}$ (lower plot)
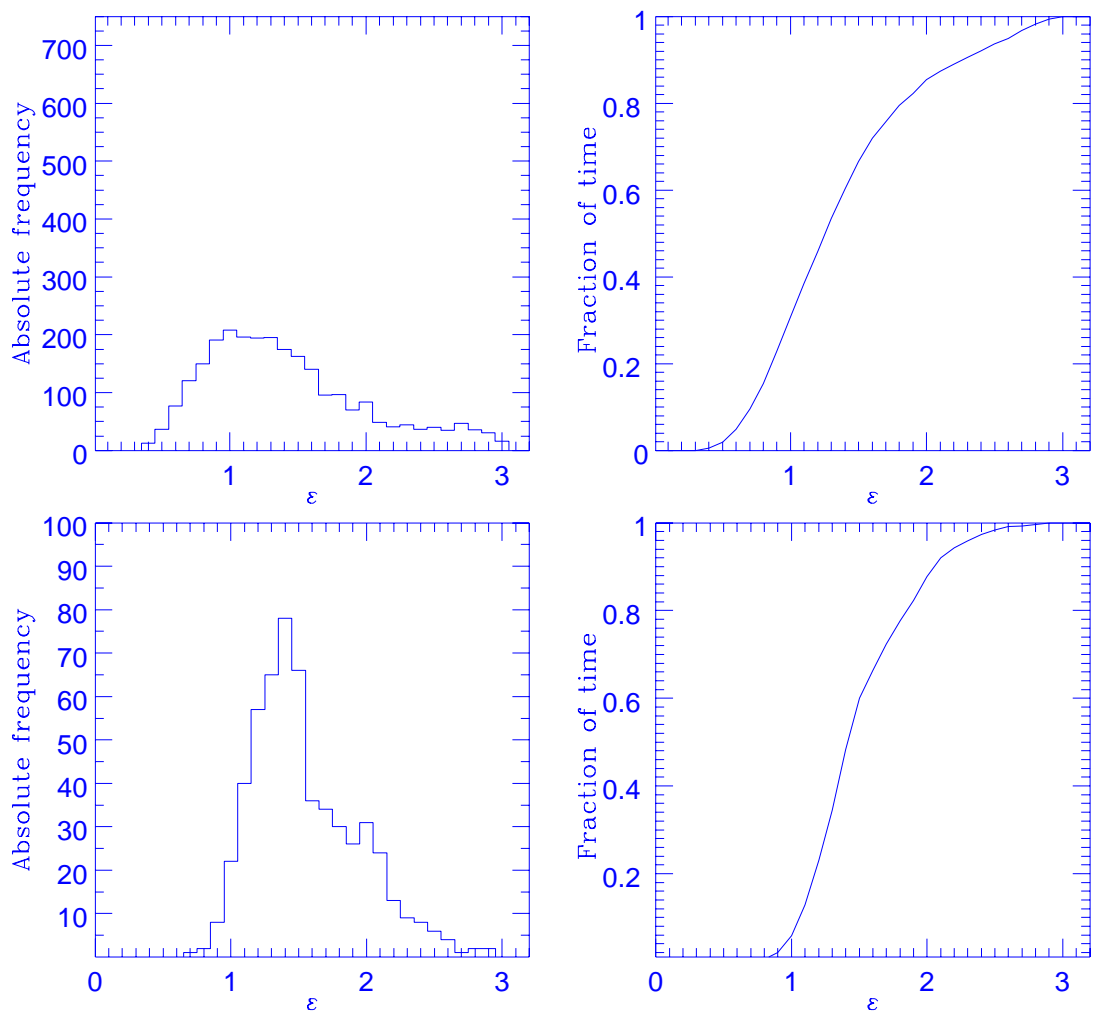

Fig. 3b). Seeing data statistics: FWHM probability distribution and associated cumulative distribution function for combined level i.e. $6-18 \mathrm{~m}$ (upper plot) and DIMM measurements (lower plot) 


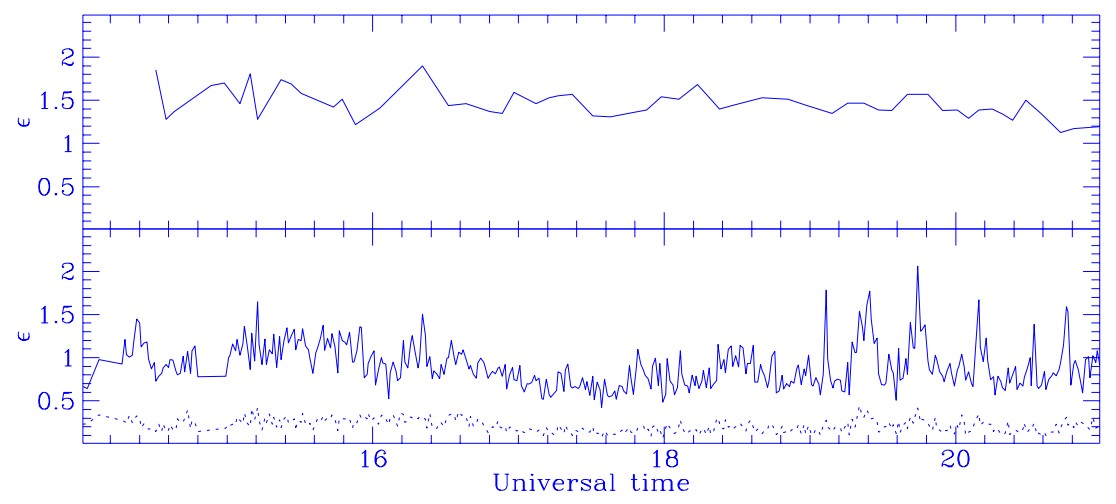

Fig. 4. (top) Seeing measurements obtained using DIMM at the focus of the $52 \mathrm{~cm}$ reflector telescope on 02 May, 1998. (bottom) Seeing contribution deduced from the microthermal measurements, solid line represents the $6-12 \mathrm{~m}$ and dots represent the $12-18 \mathrm{~m}$

Table 2. Comparison of surface layer contribution to seeing with other sites

\begin{tabular}{cccc}
\hline Site & $\begin{array}{c}\text { Height range } \\
\text { (metre) }\end{array}$ & $\begin{array}{c}\epsilon \text { (mean) } \\
\text { (arcsec) }\end{array}$ & $\begin{array}{c}\epsilon \text { (median) } \\
\text { (arcsec) }\end{array}$ \\
\hline South Pole & 7 to 17 & 0.46 & 0.42 \\
& 17 to 27 & 0.37 & 0.31 \\
& 7 to 27 & 0.64 & 0.59 \\
La Palma & 6 to 12 & 0.07 & 0.065 \\
Devasthal & 6 to 12 & 1.28 & 1.17 \\
& 12 to 18 & 0.32 & 0.30 \\
& 6 to 18 & 1.36 & 1.25 \\
\hline
\end{tabular}

assumption that the seeing is worst in the beginning of the night and improves later.

A comparison of our results for the Devasthal site with those obtained at La Palma (Vernin \& Muñoz-Tuñón 1994) and at the South Pole in Antarctica (Marks et al. 1996) is given in Table 2. The mean and median values quoted in the table for La Palma are calculated by us from the reported data for four nights by Vernin \& MuñozTuñon (1994). From the table it can be inferred that the surface layer contribution to seeing varies from site to site as it depends on the geography of the site under study.

Echevarria et al. (1998) have mentioned that the local value of the seeing is considered to be zero arcsec at a height of $\sim 100 \mathrm{~m}$ from the ground, and it increases as the starlight passes through the turbulent boundary layers of the atmosphere below $100 \mathrm{~m}$. Above $100 \mathrm{~m}$ the star image is considered to be degraded only by the turbulent layers of the free atmosphere, which mostly reside at a height of $10 \mathrm{~km}$ or so. They have also concluded that the total value of the seeing $S_{\mathrm{T}}$ is given by the relation as

$S_{\mathrm{T}}=\left(S_{\mathrm{L}}^{5 / 3}+F A^{5 / 3}\right)^{3 / 5}$

where $S_{\mathrm{L}}$ is the local value of the seeing measured for a particular slab and $F A$ is the seeing that one would observe at a height of $100 \mathrm{~m}$. We have taken the value of $F A=0.52^{\prime \prime}$ given by Cromwell et al. (1998). Adopting this value of $F A$ in Eq. (8), we estimated the total seeing $S_{\mathrm{T}}$ for the Devasthal site as $1.52^{\prime \prime}$ which is in good agreement with the value of $1.51^{\prime \prime}$ obtained from DIMM measurements. It is relevant to mention here that both DIMM and microthermal measurements were carried out simultaneously at Devasthal. Considering the seeing contribution due to the 12 to $18 \mathrm{~m}$ slab as $0.32^{\prime \prime}$ (see Table 1 ), we estimated the value of $S_{\mathrm{T}}$ as $0.65^{\prime \prime}$. This indicates that if the telescope is located at a height of $\sim 13 \mathrm{~m}$ above the ground, one can achieve sub arcsec angular resolution for a significant fraction of the observing time. This is comparable to the seeing evaluated by other investigators for various sites. If the height of the telescope is raised to $18 \mathrm{~m}$, we can attain seeing of $\sim 0.5^{\prime \prime}$.

\section{Conclusions}

It is important to know the local seeing condition as it decides the height for locating an optical telescope above the ground. The surface layers up to $12 \mathrm{~m}$ above the ground significantly deteriorates the stellar images at the Devasthal site. Its contribution due to 6 to $12 \mathrm{~m}$ slab is $\sim 1.28^{\prime \prime}$. This value reduces to $\sim 0.3^{\prime \prime}$ for the 12 to $18 \mathrm{~m}$ slab. A seeing of $0.65^{\prime \prime}$ can be achieved by putting the telescope at a height of $\sim 13 \mathrm{~m}$ above the ground. A better value for seeing $\sim 0.5^{\prime \prime}$ can be obtained, if the telescope is located above $18 \mathrm{~m}$ from the ground. Although during the last few years, enormous efforts have been made for characterizing the sites astronomically but very few observatories have the desired database. The largest database has been established by ESO at La Silla and Paranal, developing a very extensive and reliable seeing monitoring using various instruments, as well as the ORM at La Palma. It is intended to carry out both DIMM and microthermal measurements and to use All Weather Station for obtaining more information about the Devasthal site. This program will provide us a large database on both astronomical and meteorological data at Devasthal site. 
Acknowledgements. The authors are indebted to all those who helped in the recording of microthermal and DIMM measurements at Devasthal site. The discussions held during the designing stage of the instrument with Prof. S.N. Tandon are gratefully acknowledged. We also thank Mr. R.C. Pant for his valuable contribution in setting and maintaining the instrument at the site. The comments given by the referee Dr. J. Vernin are gratefully acknowledged which helped in improving the contents of the paper.

\section{References}

Barletti R., Ceppatelli G., Moroder E., Paterno L., Righini A., 1974, J. Geophys. Res. 79, 4545

Barletti R., Ceppatelli G., Paterno L., Righini A., Speroni N.J., 1976, J. Opt. Soc. Am. 66, 1380

Coulman C.E., 1969, Solar Phys. 7, 122

Coulman C.E., 1985, ARA\&A 23, 19

Cromwell R.H., Blair C.N., Woolf N.J., 1998, in Site testing for high binocular telescope, LBT Technical Memo (in preparation)

Dierickx P., 1992, J. Mod. Opt. 39, 569
Echevarria J., et al., 1998, Rev. Mex. A\&A 34, 47

Erasmus D., Andre Thompson Liard A., 1986, in: Advanced Technology Optical Telescopes III, Barr L.D. (ed.). Tucson, p. 148

Fried D.L., 1966, J. Opt. Soc. Am. 56, 1372

Hall D.N.B., 1967, Appl. Opt. 6, 1992

Hufnagel R.E., Stanley N.R., 1964, J. Opt. Soc. Am. 54, 52

Ken Knight, Gatewood G.D., Kipp S.L., Black D., 1977, A\&A L27

Lynds C.R., 1963, IAU Symp. 19, 126

Marks R.D, Vernin J., Azouit M., Briggs J.W., Burton M.G., Ashley M.C.B., Manigault J.F., 1996, A\&AS 118, 385

Muñoz-Tuñón C., Vernin J., Varela A.M., 1997, A\&AS 125, 183

Obukhov A.M., 1969, Izv. Akad. Nauk. SSSR, Ser Geograf. Geofis. 13, 58

Pant P., Sagar R., 1998, Bull. Astr. Soc. India 26, 397

Roddier F., 1981, Prog. Opt. 19, 281

Tatarski V.I., 1961, Wave Propagation in a Turbulent Medium. McGraw-Hill, New York

Vernin J., Muñoz-Tuñón C., 1992, A\&A 257, 811

Vernin J., Muñoz-Tuñón C., 1994, A\&A 284, 311 